\title{
Quantum Algorithm for Continuous Global Optimization
}

\author{
October 8, 2001 \\ V. Protopopescu and J. Barhen \\ Center for Engineering Science Advanced Research \\ Computing and Computational Sciences Directorate \\ Oak Ridge National Laboratory \\ Oak Ridge, T N 37831-6355
}

\begin{abstract}
We investigate the entwined roles of information and quantum algorithms in re ducing the complexity of the global optimization problem (GOP). We show that: (i) a modest amount of additional information is su \pm cient to map the general continuous G OP into the (discrete) G rover problem; (ii) while this additional information is actually available in some classes of GOPs, it cannot be taken advantage of within classical optimization algorithms; (iii) on the contrary, quantum algorithms o $\operatorname{Rer}$ a natural framework for the ex cient use of this information resulting in a speed-up of the solution of the GOP.
\end{abstract}




\section{Global Optimization Problem}

Optimization problems are ubiquitous and extremely consequential. The theoretical and practical interest they have generated has continued to grow from the ${ }^{-}$rst recorded instance of Queen Dido's problem [13] to present day forays in complexity theory or large scale logistics applications (see Refs. [14], [9], [8], [6], and references therein). The formulation of almost any optimization problem is deceptively simple: - $n$ d the absolute minimum (maximum) of a given function - called the objective function or functional - over the allowed range of its variables. Sometimes, the function to be optimized is not speci ed in analytic form and must be evaluated point-wise by a computer program, a physical device, or other construct. Such a black-box tool is called an oracle. Of course, the brute force approach of evaluating the function on its whole domain is either impossible - if the variables are continuous - or prohibitively expensive - if the variables are discrete, but have large ranges in high dimensional spaces. Since in general each oracle invocation (function evaluation) involves an expensive computational sequence, thenumber of function evaluations needs to be kept to a minimum. The number of invocations of the oracle measures the query complexity of the problem and gives a fair - although by no means unique - idea of its di \pm culty or $\backslash$ hardness" [5]. Therefore, the number of oracle invocations is one of the paramount criteria in comparing the e \pm ciency of competing optimization algorithms.

The primary di \pm culty in solving the GOP stems from the fact that the familiar condition for determining extrema (namely, annulment of the gradient of the objective function) is only necessary (the function may have a maximim, a minimum, or not have an extremum at all !) and local (it does not distinguish between local and global extrema). Indeed, the generic strategy to ${ }^{-}$nd the global minimum involves two main operations, namely: (i) descent to a local minimum and (ii) search for the new descent region. Usually, the former operation is deterministic and the latter stochastic. This strategy is marred by additional problems. First, descent assumes a certain degree of smoothness, which is not always warranted. When the dimensionality of the problem is large, the search of the phase space becomes more and more responsible for increasing the query complexity of the problem. Finally, after determining a local minimum, the algorithm is usually trapped in it and special operations have to be designed to restart the search. The $\backslash$ hardness" of the GOP is well illustrated by the following example for which the approach described above seems powerless. De ${ }^{-}$ne the function $\mathrm{f}:[0 ; 1]$ ! f $0 ; 1 \mathrm{~g}$ as follows:

$$
f(x)=\begin{array}{cc}
8 & 1 \text { for } 0 \cdot x \cdot a i^{2}=2 \\
& 0 \text { for } a i^{2}=2<x<a+{ }^{2}=2 \\
1 \text { for } a+{ }^{2}=2 \cdot x \cdot 1:
\end{array}
$$

where a $2\left({ }^{2}=2 ; 1 i^{2}=2\right)$. To obtain the minimum of this function, one should evaluate it within the ${ }^{2}$ interval around the unknown number $a$. If this function is de ned like an oracle (i.e., if one does not know the position of the point a), the probability of choosing 
an $\mathrm{x}$ within this interval is ${ }^{2}$. For the $\mathrm{n}$-dimensional version of this oracle, the probability becomes ${ }^{2 n}$, and the complexity of the problem grows exponentially with $n$ (the dimensionality curse). Of course, this is an extreme case, for which knowledge about the derivatives (they are all zero whenever de ned !) would not help. This and related issues have been deftly discussed by Wolpert and Macready in connection with their $\backslash$ No Free Lunch" (NFL) theorem [15].

In the light of the previous example, it seems that without additional knowledge about the structure of the function there is no hope to decide upon an intel ligent optimization strategy and one is left with either strategies that have limited albeit e \pm cient applicability or the exhaustive search option.

Thus, new approaches are needed to reduce the complexity of the problem to manageable complexity. Recently, quantum computing has been hailed as the possible solution to some of the computationally hard classical problems [11]. Indeed, Grover's [7] and Shor's [12] algoritms provide such solutions to the problems of " nding a given element in an unsorted set and the prime factorization of very large numbers, respectively. Here we present a solution to the continuous G OP in polynomial time, by developing a generalization of Grover's algorithm to continuous problems. This gener al ization requires additional information on the objective function. In many optimization problems, some of this additional information is available (see below). W hile other required information may be more di \pm cult to obtain in practical applications, it is important to understand - from a theoretical point of view - the role of the information in connection to the di \pm culty of the problem, and to be able to assess a priori what various information is relevant and for what. For instance, if the objective function were an analytic function, the knowledge of all its derivatives at a given point would allow, in principle, the \knowledge" of the function every where else in the domain of analyticity. However, to actually 'nd the global minimum, the function would still have to be calculated everywhere ! In other words, the (additional) knowledge of all the derivatives at a given point cannot be et ciently used to locate the global minimum, although in principle it is equivalent to the knowledge of the function at all points. In fact, to locate the global minimum, both methods would require exhaustive calculations.

\section{Grover Quantum Algorithm}

A quantum computation is a sequence of unitary transformations on the initial state of the wave function, $\tilde{A}$. As such, quantum computation is purely deterministic and reversible. It requires the initialization or preparation of the initial state, the actual \computation" and the read out of the result eßected through a measurement of the "nal state. If the algorithm is e \pm cient, then, with probability (much) higher than $1=2$, the measurement would collapse the "nal state onto the desired result. Computer architectures needed to implement classical or quantum algorithm are realized in terms of gates. As opposed to classical gates that operate on bits taking values in the set $\mathrm{f} 0,1 \mathrm{~g}$, quantum gates operate on normalized vectors in a ${ }^{-}$nite-dimensional complex Euclidian space. In principle, any 
quantum computer can be viewed as an assembly of elementary quantum gates, such as the NOT and CNOT gates. The NOT gate is the $2 \mathrm{f} 2$ matrix $\begin{array}{lll}0 & 1 \\ 1 & 0\end{array}$. It acts on a qubit, $\mathrm{q}$, which is the normalized state in a two dimensional Euclidian space, $\mathbb{C}^{2}$ :

$$
q=\otimes j 0>+{ }^{-} j 1>; \quad j \circledast j^{2}+j^{-} j^{2}=1 ;
$$

by exchanging the level populations. The CNOT gate acts on four dimensional vectors in $\mathbb{C}^{4}$. Obviously, some of these vectors can be represented as a tensor product of two two-dimensional vectors; however other vectors in $\mathbb{C}^{4}$ cannot be written in this form. These latter states are called entangled states and play a crucial role in quantum algorithms [11]. Quantum algorithms are (i) intrinsically parallel and (ii) yield probabilistic results. These properties re ect the facts that: (i) the wave function, $\tilde{\mathrm{A}}$, is nonlocal and, in fact, ubiquituous and (ii) the quantity $\mathrm{j} \tilde{\mathrm{A}} \mathrm{j}^{2}$ is interpreted as a probability density.

Grover's original algorithm provides a solution to the following problem. Suppose we have a set of $\mathrm{N}$ unsorted objects, $E=f x_{1} ; x_{2} ;:: x_{N} g$, and an oracle function $f: E$ ! $f 0 ; 1 g$, such that $f\left(x_{1}\right)=1$ and $f\left(x_{i}\right)=0, i=2 ;:: N$ : U sing the oracle, "nd the element $x_{1}$ in the unsorted set $\mathrm{E}$.

On average, the classical solution will involve $\gg N=2 \gg O(N)$ evaluations of the oracle. The quantum algorithm proposed by Grover [7] reduces this number to $O\left({ }^{N}\right)$. In a generalized version of the problem, there may be $L$ I special " elements for which the oracle returns the value one; then the number of evaluations required to ${ }^{-}$nd one of them is of the order $\mathrm{O}(\overline{\mathrm{N}=\mathrm{L}})$.

We give a brief presentation of G rover's quantum algorithm [7]. First, we identify the set $E$ with the complex E uclidean space $\mathbb{C}^{N}$ and the elements $x_{i} 2 E$ with the unit vectors in $\mathbb{C}^{N},\left\langle x_{i} j x_{j}>= \pm_{j}\right.$, where $\pm_{j}$ is the K ronecker symbol. Then construct the normalized average state of all the elements $j x_{i}>$ :

$$
j w>=p_{\bar{N}}^{1} x_{i=1}^{N} j x_{i}>=p_{\bar{N}}^{1} j x_{1}>+\frac{p}{p_{\bar{N}}{ }_{\bar{N}}^{1}} j x_{i}>\text { : }
$$

In the second representation, the unit vectors orthogonal to $\mathrm{j}_{1}>$ are lumped together in the unit vector $\mathrm{jx}$ ? $>$, which formally reduces the problem to a bidimensional space and simpli ${ }^{-}$es the presentation and interpretation of the algorithm.

We note that the scalar product $\left\langle x_{1} j \mathrm{w}\right\rangle=p \frac{1}{\bar{N}}:=\cos ^{-}=\sin \circledast$ where ${ }^{-}$denotes the angle between the vectors $\mathrm{jw}>$ and $\mathrm{jx} \mathrm{x}_{1}>$ and $\circledast$ denotes its complement, i.e. the angle between the vectors $j w>$ and $j x_{1}^{?}>$.

The construction of the state $j w>$ can be done in $\log _{2} N=n$ steps by applying (in parallel) $\mathrm{n}$ Hadamard transformations, $\mathrm{H}=\frac{1}{\overline{2}} \quad \begin{array}{ll}1 & 1 \\ 1 & \mathrm{i}\end{array}$ on the initial zero state, $j 0>-\ldots: \ldots ;-j 0>$. In the $\mathrm{fjx}_{1}>; j \mathrm{x}_{1}^{?}>\mathrm{g}$ basis, we construct the operators:

$\mathrm{n}$ times 


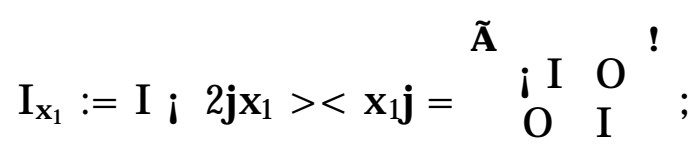

which executes a re ection (sign inversion) of the $\mathrm{x}_{1}$-component of a vector and

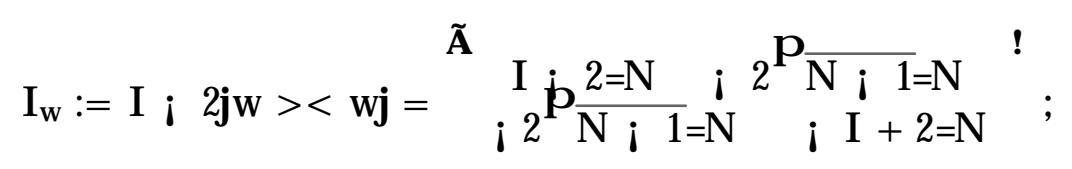

which represents a re ection (sign inversion) of the w-component of a vector. At thelevel of the oracle, the operator $I_{x_{1}}$ is implemented by $(i 1)^{f(:)}(:)$, which does not depend explicitly on the unknown element $x_{1}$, while the application of the operator $I_{w}$ is obvious, since the average state is known. We de ne now the amplitude ampli ${ }^{-}$cation operator:

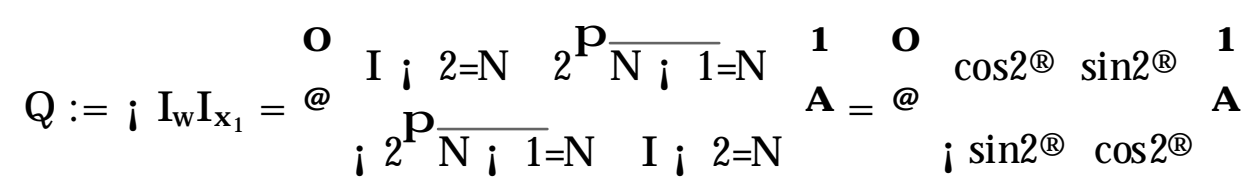

which, in the compressed, two-dimensional representation of the problem, represents a rotation of the state vector with an angle 2 @ towards $j x_{1}>$. This means that each application of the operator $Q$ will increase the weight of the unknown vector $j x_{j}>$ (which explains

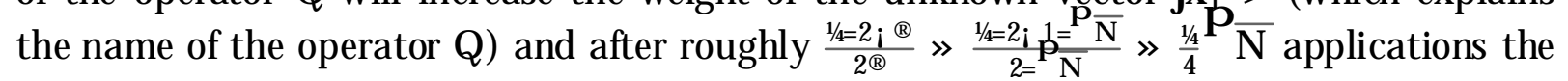
state vector will be essentially parallel to $\mathrm{jx} \mathrm{x}_{1}>$, whereupon a measurement of the state will yield the result $j \mathrm{x}_{1}>$ with a probability very close to unity. We mention that for (and only for) $\mathrm{N}=4$, the result is obtained with certainty, after only one application. In general, if one continues the application of $Q$, the state vector continues its rotation and the weight of $\mathrm{jx}_{1}>$ decreases; eventually, the evolution of the state is cyclic as prescribed by the unitary evolution. In the original, $\mathrm{N}$-dimensional representation, the operator $\mathrm{I}_{\mathrm{w}}$ has the representation:

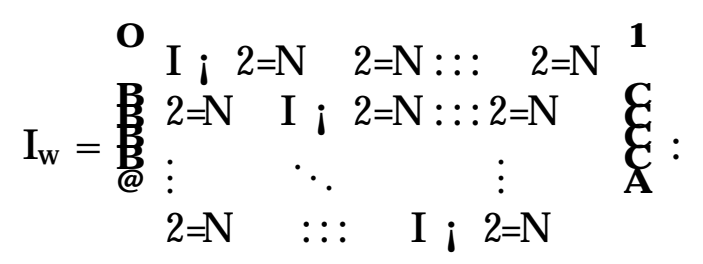

Using this representation, it is easy to show explicitly that the algorithm can be implemented as a sequence of local operations such as rotations, Hadamard transforms, etc. [7]

It is easy to check that if the oracle returns the same value for all the elements, i.e. there is no \special" element in the set $\mathrm{E}$, the ampli ${ }^{-}$cation operator $\mathrm{Q}$ reduces to the identity operator I and, after the required number of applications, the measurement will return any of the states with the same probability, namely $1=\mathrm{N}$. In other words, the algorithm behaves consistently. 


\section{Solution of the Continuous Global Optimization Problem}

Grover's algorithm has been applied previously to a discrete optimization problem, namely - nding the minimum among an unsorted set of $\mathrm{N}$ di ßerent objects. Därr and Hoyer [4] adapted Grover's original algorithm and solved this problem with probability strictly greater than $1=2$ using $O\left({ }^{N}\right)$ function evaluations (oracle invocations).

In this article, we map the continuous GOP to the Grover problem. Once this is achjeved, one can apply either Grover's al gorithm and obtain an almost certain result with $O\left({ }_{N}\right)$ function evaluations. However, the mapping of the GOP to Grover's problem is not automatic, but requires additional information.

B efore spelling out the required information, let us revisit the "pathological" example (1). W ithout loss of general ity, we can take ${ }^{2}=1=\mathrm{N}$ and divide the segment $[0 ; 1]$ into $\mathrm{N}$ equal intervals. By evaluating the function at the midpoint of the $\mathrm{N}$ intervals, we obtain a discrete function that is equal to 1 in $\mathrm{N}$ i 1 points and equal to 0 in one point, which - up to an unessential transformation - is equivalent to G rover's problem. Direct application of any Grover-like al gorithm yiel ds the corresponding result. Of course, generalization to any dimensionality $d$ is trivial. A problem that seemed intractable classically becomes much easier within the quantum computing framework. We shall return to this example after discussing the general case

Consider now a real function of $d$ variables, $f\left(x_{1} ; x_{2} ;: ;: ; x_{d}\right)$. W ithout restricting generality, we can assume that $f$ is de ned on $[0 ; 1]^{d}$ and takes values in $[0 ; 1]$. A ssume now that: (i) there is a unique global minimum which is reached at zero; (ii) there are no local minima whose value is in "nitesimally close to zero; in other words, the values of the other minima are larger than a constant $\pm>0$, and (iii) the size of the basin of attraction for the global minimum measured at height \pm is known; we shall denote it $\phi$.

Then our implementation paradigm is the following: (i) instead of $f(:)$, consider the transformation $g(:):=(f(:))^{1=m}$. For su \pm ciently large $m$, this function will take values very close to one, except in the vicinity of the global minimum, which will maintain its original value, namely zero. Of course, other transformations can be used to achieve essentially the same result. We calculate $m$ such that $\underline{1}^{1}=\mathrm{m}=1=2$. To avoid technical complications that would not change the tenure and conclusions of the argument, we assume that $\phi=1 \mathrm{AM}$ where $M$ is a natural number, and divide the hypercube $[0 ; 1]^{d}$ in small d-dimensional hypercubes with sides $\phi$. At the midpoint of each of these hypercubes, de ne the function $h(x):=\operatorname{int}[g(:)+1=2]$ (here int denotes the integer part). The function $h(:)$ is de- ned on a discrete set of $\mathrm{N}$ points, $\mathrm{N}=\mathrm{M}$, and takes only values one and zero; by our choice of constants, the region on which it takes value zero is a hypercube with side $\phi$. Thus we have reduced the problem to the Grover setting. A pplication of Grover's algorithm to the function $h(:)$, will result in a point that returns the value zero; by construction, this point belongs to the basin of attraction of the global minimum. We return then to the original function $f(:)$ and apply the descent technique of choice that will lead to the global minimum. If the basin of attraction of the global minimum is narrow, the 
gradients of the function $f(:)$ may reach very large values which may cause overshots. Once that phase of the algorithm is reached, one can proceed to apply a scaling (dilation) transformation that maintains the descent mode but moderates the gradients. On the other hand, as one approaches the global minimum, the gradients become very small and certain acceleration techniques based on non-Lipschitzian dynamics may be required [1, 2]. If the global minimum is attained at the boundary of the domain, the algorithm above will - nd it without additional complications.

\section{Practical Implementation Considerations}

It is clear that, in general, the conditions imposed on the functions $f(:)$ are rather strong, su \pm cient conditions. However: (a) these conditions are both satis ${ }^{-}$ed and explicitly given for the academic Igolf course" example mentioned before and (b) while they do not help reduce the complexity of the classical descent/ search algorithm, they make a remarkable di eerence in the quantum framework.

In fact, assumption (i) is satis ${ }^{-}$ed by a large class of important practical problems, namely parameter identi ${ }^{-}$cation encountered e.g. in remote sensing, pattern recognition, and, in general, inverse problems. In these problems the absolute minimum, namely zero, is attained for the correct values of the parameters, matching of patterns, and ${ }^{-}$tting of output to input. A ssumption (i) can be relaxed in the sense that the function may have multiple global minima, all equal to zero. Functions with multiple global minima will simply result in Grover problems with multiple \special" elements and can be treated accordingly if the number of global minima is known.

A ssumption (ii) can be replaced with the much more reasonable assumption that $f$ has a - nite number of local minima. This would prevent the value of any local minimum to be in ${ }^{-}$nitesimally close to the value of the global minimum.

Assumption (iii) is the most di \pm cult to ful ${ }^{-} \|$in practical problems. However, this assumption could al so be relaxed with no signi ${ }^{-}$cant performance loss if more e \pm cient (e.g. exponentially fast) unstructured search quantum algorithms were available [10]. For the time being, the likelihood of exponentially fast search algorithms is uncertain.

Recently, Chen and Diao [3], proposed an algorithm which was supposed to achieve an exponential (as opposed to polynomial) speed-up of the unstructured search. Unfortunately, subtle complexity hidden in one of the proposed steps makes this algorithm unusuitable for very fast search.

Despite their scarcity and still el usive implementation in a practical quantum computer, quantum algorithms could bring very promising solutions to hard computational problems. It seems likely that - like the algorithms prop osed so far - future quantum algorithms will be much more $\mid$ problem tailored" than their classical counterparts. Therefore, speci ${ }^{-} c$ additional information is crucial. In general, this information may be di \pm cult to obtain, but - as illustrated above - its bene ${ }^{-}$ts may signi ${ }^{-}$cantly outweigh its cost. Indeed, for very high dimensional, computationally intensive problems, even the polynomial reduction of complexity o ßered by the Grover algorithm is extremely signi ${ }^{-}$cant. 


\section{Acknowledgments}

This work was partially supported by the $M$ aterial Sciences and Engineering Division Program of the DOE O \pm ce of Science under contract DE-AC05-000R22725 with UTB attelle, LLC. We thank Drs. R obert P rice and I ran Thomas from DOE for their support. V. P. thanks Dr. Cassius D'H elon for an enlightening discussion on Ref. 3 and for a careful reading of the manuscript.

\section{References}

[1] J . Barhen and V. Protopopescu, Generalized TRUST A lgorithm for G lobal Optimization, State of the Art in Global Optimization, C.A. Floudas and P.M. Pardalos eds., pp. 163-180, K luwer A cademic Press, Dordrecht, B oston 1996.

[2] J . Barhen, V. Protopopescu, and D. Reister, TRUST: A Deterministic Algorithm for Global O ptimization, Science 276, 1094-1097 (1997).

[3] G. Chen and Z. Diao, Exponentially Fast Quantum Search Algorithm, quant$\mathrm{ph} / 0011109$.

[4] C. DÄrr and P. Hoyer, A Quantum Algorithm for Finding the M inimum, quant$\mathrm{ph} / 9607014$

[5] H. Lydia Deng and J. A. Scales, Characterizing Complexity in Generic Optimization Problems, Preprint, center of Wave phenomena, Golden, Colorado, CW P-208P, October 1996.

[6] C. A. Floudas and P. M. Pardalos, eds. State of the Art in Global Optimization: Computational Methods and Applications, Kluwer Academic Publishers, Dordrecht, Boston, 1996.

[7] L. K. G rover, Q uantum M echanics Helps in Searching a Needle in a Haystack, Phys. Rev. Lett 78, 325-328 (1997).

[8] W. W. Hager, D. W. Hearn, and P. M. Pardalos, eds. Large Scale O ptimization: State of the Art, K luwer A cademic P ublishers, Dordrecht, Boston, 1994.

[9] R. Horst and H. Tuy, Global Optimization, 2d ed., Springer-Verlag, Berlin, 1993.

[10] V. Protopopescu and J. Barhen, to be published.

[11] M. Nielsen and I. Chuang, Quantm Computation and Quantum Information, Cambridge University Press, Cambridge, UK, 2000; A . O. Pittenger, An Intyroduction to Q uantum Computing Algorithms, Birkhäuser, B oston, 2000. 
[12] P. Shor, Algorithms for Quantum Computation: Discrete Logarithms and Factoring, P roceedings of the $35^{\text {th }}$ A nnual Symposium on Foundations of Computer Science, 1994, p. 124-134.

[13] D. R. Smith, Variational Methods in Optimization, Prentice Hall, Inc., Englewood Clißs, N.J ., 1974.

[14] A. Törn and A. Zilinskas, Global Optimization, Springer-Verlag, Berlin, 1989.

[15] D. H. Wolpert and W. G. M acready, No Free L unch Theorems for Optimization, IE E E Trans. on Evolutionary Computing, $167\{82$ (1997); see also W. G. Macready and D. H. Wolpert, What Makes an Optimization Problem Hard ?, Complexity, 5, 40\{46 (1996). 